\section{$\underset{\substack{\text { hommes } \\ \text { \& migrations }}}{ }$}

Hommes \& migrations

Revue française de référence sur les dynamiques

migratoires

1302 | 2013

Le Japon, pays d'immigration?

\title{
Les caractéristiques de la xénophobie au Japon
}

\section{Myungsoo Kim}

Traducteur : Chikako Mori et Hélène Le Bail

\section{CpenEdition}

Journals

Édition électronique

URL : http://journals.openedition.org/hommesmigrations/2474

DOI : $10.4000 /$ hommesmigrations. 2474

ISSN : 2262-3353

Éditeur

Musée national de l'histoire de l'immigration

Édition imprimée

Date de publication : 1 avril 2013

Pagination : 89-97

ISBN : 978-2-919040-22-3

ISSN : 1142-852X

Référence électronique

Myungsoo Kim, «Les caractéristiques de la xénophobie au Japon», Hommes \& migrations [En ligne], 1302 | 2013, mis en ligne le 31 décembre 2015, consulté le 19 avril 2019. URL : http://

journals.openedition.org/hommesmigrations/2474; DOI : 10.4000/hommesmigrations.2474 


\title{
LES CARACTÉRISTIQUES DE LA XÉNOPHOBIE AU JAPON
}

par MYUNGSOO KIM, professeur de sociologie, Kwansei Gakuin University

\author{
Comme dans la plupart des pays européens, la persistance \\ de réflexes nationalistes et xénophobes s'observe \\ dans l'archipel japonais, notamment à l'égard des Coréens. \\ Le sens donné à la xénophobie dans une société qui \\ promeut une définition mono-ethnique de sa nation diffère \\ par exemple du cas français. Toutefois, certains éléments \\ se retrouvent : histoire coloniale, avancée du néolibéralisme \\ et nouvelles immigrations.
}

\section{Une enquête sur les spécificités locales des sentiments xénophobes}

Au cours des vingt dernières années, l'intérêt scientifique porté à la xénophobie s'est développé de façon remarquable à travers le monde. Ainsi deux observations sont largement partagées : premièrement, la xénophobie se développe en réaction à l'incertitude économique et aux transformations des systèmes sociaux dues à la mondialisation. Les mutations structurelles macro échappent aux capacités d'analyse des hommes et le migrant, considéré comme la cause de la détérioration des conditions de vie, devient un bouc émissaire. Ce processus, amplifié par les discours anti-immigrés véhiculés par les médias et les partis d'extrême droite, aboutit à une montée de la xénophobie de plus en plus visible dans divers pays. Ensuite, au-delà des données comparatives internationales, tel Eurobarometer Survey, qui proposent des mesures de la xénophobie, les recherches empiriques ont permis de mettre en évidence que les mouvements xénophobes émergent et se développent indépendamment du contexte historique de chaque pays. Cela a permis également d'identifier plusieurs sources possibles des sentiments xénophobes ${ }^{1}$.

Aussi excellentes que puissent être les explications en termes de mondialisation, les comparaisons de données internationales ou la compréhension des 
phénomènes transnationaux, il est bien plus difficile de saisir la réalité locale de chaque pays. Il existe donc un risque réel de sous-estimer, voire de négliger, la particularité de certains pays à force de se focaliser exclusivement sur les causes communes qui s'observent dans nombre de pays, tels les changements économiques ou l'augmentation de l'immigration.

Pour comprendre la xénophobie localement, au Japon, il faut saisir ses fondements historiques. La xénophobie dans ce pays a la particularité de reposer, d'une part, sur l'absence de distinction entre "étranger" et "immigré", et, d'autre part, sur un discours de "la menace de l'ordre public" issu des mouvements d'indépendance des colonies. Par ailleurs, le discours du "poids économique" de l'immigration s'est peu développé et la notion d'assimilation a, au Japon, une histoire singulière.

Une enquête menée au niveau d'une ville permet de saisir les particularités de cette xénophobie à travers la façon dont les Japonais perçoivent leur société et ses membres. Ainsi, au Japon, la xénophobie se construit au moins sur quatre principales sources de sentiments xénophobes.

\section{Absence de distinction entre "étranger" et "immigré"}

Au Japon, on ne parle jamais d'“immigrés" pour désigner les personnes de nationalité étrangère résidant depuis longtemps dans le pays ${ }^{2}$.

Par exemple, les personnes de nationalité étrangère qui sont nées et ont été élevées au Japon sont le plus souvent légalement et socialement traitées comme des étrangers. Cela est vrai pour les droits définis par la loi avec une clause de nationalité, tel le droit de vote. Au-delà, il n'est pas rare que les étrangers n'aient pas le droit de devenir membres d'associations locales ou d'associations de résidents ; les discriminations indirectes sont aussi nombreuses, tel l'accès au logement.

Cela ne concerne pas seulement les étrangers en séjour court. C'est aussi le cas pour les Coréens ou les Taïwanais arrivés après la colonisation japonaise de leur pays. Certaines familles sont là depuis un siècle et aujourd'hui nombreux sont les descendants de la quatrième génération. Pour diverses raisons historiques, parmi eux, 400000 environ ont conservé leur nationalité et sont traités comme de simples "étrangers". Dans les années 1980, afin de se distinguer des résidents étrangers à court terme, des Coréens du Japon ont proposé l'expression "étrangers établis" (teijû gaikokujin), les médias ne l'ont pas adoptée et bien que le nombre d'étrangers établis au Japon à long terme augmente (voir graphique 1), l'occurrence de cette expression diminue ${ }^{4}$.

Autrement dit, lorsque l'on veut exprimer l'appartenance à la société japonaise, il n'existe que deux catégories : “Japonais" ou "étranger", les concepts intermédiaires du type "denizen", ou même la reconnaissance de la présence d'immigrés" ne sont pas utilisés au Japon.

Même une personne qui a obtenu la nationalité japonaise, et qui, vue de l'extérieur, ne peut en rien être distinguée d'un Japonais, ne sera pas systématiquement reconnue comme "un Japonais authentique" par la société. Avoir des origines étrangères, même invisibles, peut être perçu négativement. C'est ce qu'a très bien compris Shintaro Ishihara ${ }^{5}$ lorsque, en 1983, pendant la campagne électorale législative, son secrétaire a mené une campagne électorale particulièrement polémique contre le candidat Shôkei Arai ${ }^{6}$, adversaire de M. Ishihara dans la même circonscription : il s'agissait de coller sur chaque affiche du candidat Arai des autocollants noirs indiquant "Coréen du Nord naturalisé

2. Quand les journaux japonais utilisent le terme “immigré”, c'est dans la majorité des cas pour parler d'autres pays. Aucune distinction particulière n'est faite pour les personnes ayant pris la nationalité du pays d'accueil ou pour les descendants nés dans le pays d'accueil. 3. Plus précisément, depuis 1991, ils bénéficient d'un statut de résidence relativement stable : le "statut de résident permanent spécial". 4. Selon la base de données du journal Asahi, la fréquence du terme dans les articles recule dans les années 2000. Dans les années 1990, l'expression était souvent utilisée dans des articles abordant le débat sur le droit de vote local pour les Coréens du Japon. Après le tournant à droite du début des années 2000 , la question de la participation politique locale des étrangers est en recul. 5. Ishihara Shintarô, gouverneur de Tokyo jusqu'en automne 2012, est très connu pour ses positions anti-immigrés et ses discours discriminants à l'égard des anciens colonisés (note des traductrices). 
en 1966" pour souligner qu'il n'était pas un "vrai Japonais"...

De même, le président de la célèbre société Soft Bank, Masayoshi Son, est né et a grandi au Japon, a pris la nationalité japonaise, mais reste encore aujourd'hui sur Internet la cible d'interjections haineuses qui le qualifient de "Coréen". On peut lire par exemple : "Son Masayoshi est le fils d'un bandit coréen entré au Japon clandestinement après la guerre. Il a d'abord fabriqué de l'alcool clandestin, puis il a travaillé dans le secteur financier souterrain, dans les pachinko, et a construit une fortune sur ces activités criminelles. Lui-même méprise le Japon et accorde ses faveurs aux Coréens du Japon et à la Corée." Ces rumeurs ne sont fondées sur aucun fait réel ${ }^{7}$. La difficulté à être reconnu comme Japonais une fois naturalisé ne touche pas seulement les Coréens. Ren Hou (Lien Fang), députée à la Diète, deux fois ministre, parce que son père est d'origine taïwanaise, a été récemment victime de fausses déclarations mettant en cause son attachement au Japon.

Ce que montrent ces exemples, c'est que l'identité ethnique ou la nationalité importe peu. À moins de pouvoir prouver que l'on est un "Japonais" traditionnel, on reste traité comme un étranger et un citoyen de second ordre. Le mot "étranger" s'applique bien sûr aux résidents de nationalité étrangère, mais il est aussi souvent compris dans un sens plus large pour désigner les minorités ethniques (populations autochtones inclues).

\section{Le discours de la "menace à l'ordre public"}

C'est l'histoire et le contexte social d'un pays qui expliquent la prédominance d'un type de discours xénophobe. Dans le cas du Japon, il est fondé sur la "menace à l'ordre public", avec l'idée que l'augmentation de la population étrangère fait monter la criminalité" ou encore que les "étrangers
Figure 1 : Évolution du nombre de résidents étrangers au Japon par nationalité, 1947-2007

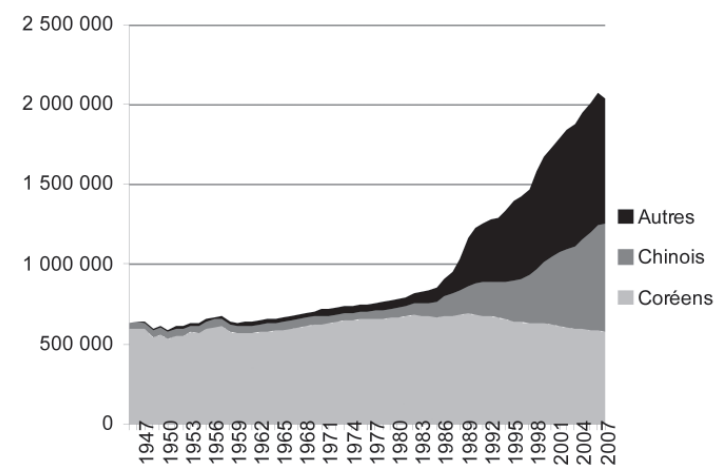

Source : Statistiques du Bureau d'immigration du ministère de la Justice, 2008 Immigration Control, en ligne URL www.moj.go.jp/nyuukokukanri/kouhou/nyukan_nyukan42.html.

ne respectent pas le tri des poubelles", etc. Mais l'idée que les étrangers sont un "poids économique", par exemple qu'ils "profitent du système de protection sociale" ou qu'ils "prennent les emplois des Japonais", n'est pas tellement exploitée, peutêtre parce qu'ils sont peu nombreux (moins de $2 \%$ de la population).

Selon les travaux récents de l'historien Masaru Tonomura, il faut chercher l'origine de ce discours sur la "menace à l'ordre public" dans les mouvements indépendantistes de la Corée. Ses principaux arguments sont les suivants. Dès 1880, quand le Japon commence à exercer son influence sur la péninsule coréenne, on observe que la presse commence aussi à établir une équation entre "émeutier" et "Coréen". Le terme d'"émeutier" dans les articles a été systématique utilisé pour parler des Coréens et à l'époque des luttes antijaponaises se sont développés les récits du "Coréen dangereux". De 1910 à la première moitié des années 1920, s'est imposée l'expression "Coréen terroriste", qui a contribué à nourrir l'image d'un "Coréen mettant en danger 
le Japon", par exemple au moment du mouvement indépendantiste du $1^{\text {er }}$ mars 1919. En 1931, quand la guerre sino-japonaise éclate, le gouvernement japonais, poussé par la nécessité de faire participer les Coréens à l'effort de production, a plutôt développé un discours sur les "efforts des Coréens pour s'assimiler". Toutefois, cela s'entremêlait avec des articles appelant à la vigilance, les autorités ellesmêmes restaient méfiantes envers les Coréens, et la population continuait de les considérer comme une menace.

Après la décolonisation, la tendance à considérer les Coréens comme un "problème pour l'ordre public" n'a pas fondamentalement évolué 8 . La gestion des Coréens a été confiée à d'anciens membres de la police politique (police responsable des colonies). Jusqu'à la fin des années 1980, la police japonaise traita les résidents coréens comme de potentiels délinquants. À partir des années 1990, les ressortissants de la Corée du Nord devinrent la seule cible des suspicions et ceci jusqu'à aujourd'hui encore.

Apparu à une époque où les étrangers étaient avant tout des Coréens issus des colonies, le discours fondé sur l'image d'un étranger source de désordre, d'indiscipline n'a pas changé, même si la population étrangère s'est diversifiée après 1980. L’image de létranger est forgée sur celle du Coréen au Japon? Selon l'enquête présentée ci-dessous, à la question "Quelle est la part des étrangers dans la criminalité au Japon ?”, la réponse médiane était $10 \%$ et la moyenne des réponses 14,9\%. En réalité, la criminalité des étrangers représente moins de $2 \%$ de la criminalité au Japon.

\section{La faible prégnance du discours du "poids économique"}

Les arguments xénophobes d'ordre économique, on l'a dit, n'ont pas tellement d'impact. Cela s'explique à nouveau par l'histoire des Coréens du Japon qui, jusqu'au milieu des années 1980, représentent la majorité des étrangers. Étant donné leur très forte discrimination et leur exclusion du marché du travail, les Coréens se sont orientés vers l'entrepreneuriat (tableau 1). Pourtant, si l'on regarde les principaux indices de réussite sociale (le nombre d'années de formation, le prestige des professions ${ }^{10}$ et le revenu), on n'observe pas de différences entre Japonais et Coréens du Japon. La réussite sociale des Coréens serait même plus élevée en moyenne (tableau 2). Ils ont surmonté les inégalités par leurs propres moyens. Pour le dire autrement, face aux discriminations à l'emploi, les Coréens du Japon ne sont pas entrés dans un rapport de compétition sur le marché du travail. Ils ont réussi à trouver une situation stable par leur propre entrepreneuriat et ne sont pas devenus un poids pour l'Étatprovidence. On peut penser que leur insertion professionnelle explique que l'argument du poids économique ne soit pas présent au Japon.

\section{Le sens donné à l'assimilation au Japon}

Le discours sur l'assimilation au Japon n'a évidemment pas pris le même sens qu'en Europe ou en Amérique ${ }^{11}$. Alors qu'il était Premier ministre en 1986, Yasuhiro Nakasone, connu pour ses

\footnotetext{
8. Myungsoo Kim, "Ethnic stratification and inter-generational differences in Japan : A comparative study of Korean and Japanese status attainment", in International Journal of Japanese Sociology, vol. 12, 2003, pp. 6-16; Yasuaki Onuma, Tanitsu minzoku shakaï no shinwa o koete. Zaïnichi kankoku chôsenjin to shutsunyû koku kanri taisei (Au-delà du mythe de la société ethniquement homogène : les Coréens au Japon et les politiques de l'immigration), Tokyo, Tôshindô, 1986. 9. Alors qu'aujourd'hui, la composition des étrangers au Japon a beaucoup évolué, comparée à l'époque coloniale, et qu'un nombre non négligeable de Japonais éprouvent un choc en faisant face à d'autres étrangers culturellement très différents, il est indispensable d'observer avec attention ce qui se passe dans les zones où il y a de nombreux newcomers. 10. Le prestige des professions signifie l'estime que les membres d'une société peuvent avoir d'une profession ou d'un métier. La classification du prestige des professions occupe une place importante dans les recherches sur les stratifications sociales au Japon. 11. Richey Sean, "Assimilation and national myths : Attitudes towards immigration in Japan", paper presented at the annual meeting of the Western Political Science Association, Manchester Hyatt, San Diego, California, mars 2008.
} 
Tableau 1. Comparaison des catégories d'emplois des Japonais et des Coréens du Japon

\begin{tabular}{|c|c|c|c|c|}
\hline \multirow[b]{2}{*}{ Col blanc supérieur } & \multicolumn{2}{|c|}{ Japonais } & \multicolumn{2}{|c|}{ Coréens du Japon } \\
\hline & 203 & $(18,6)$ & 96 & $(14,2)$ \\
\hline Col blanc inférieur & 259 & $(23,7)$ & 84 & $(12,4)$ \\
\hline Entrepreneur & 253 & $(23,2)$ & 352 & $(52,1)$ \\
\hline Col bleu & 313 & $(28,7)$ & 142 & $(21,0)$ \\
\hline Agriculteur & 64 & $(5,9)$ & 2 & $(0,3)$ \\
\hline Total & 1092 & $(100,0)$ & 676 & $(100,0)$ \\
\hline
\end{tabular}

Tableau 2. Comparaison d'indicateurs de statut, Japonais et Coréens du Japon

\begin{tabular}{|c|c|c|c|c|}
\hline & \multicolumn{2}{|c|}{ Japonais } & \multicolumn{2}{|c|}{ Coréens du Japon } \\
\hline & Moyenne & Écart type & Moyenne & Écart type \\
\hline Durée d'études & 12,35 & 2,78 & 12,01 & 2,99 \\
\hline Prestige de l'emploi & 47,32 & 11,39 & 48,02 & 12,82 \\
\hline Revenu 10000 yens & 528.79 & 252,43 & 572,69 & 276,37 \\
\hline
\end{tabular}

Source : réalisé par l'auteur à partir de deux enquêtes. D'une part, la Zainichi Kankokujin no Shakaiseiso to Shakaiishiki Zenkoku Chosa (National Survey of Social Stratification and Social Consciousness among South Koreans in Japan), 1995, et, d'autre part, l'enquête Social Stratification and Socia Mobility réalisée la même année (1995 SSM Research Series, University of Tokyo) ${ }^{12}$.

positions de droite, avait déclaré : "Les États-Unis étant un pays multi-ethnique, il est difficile d'assurer un bon niveau d'éducation. Les Noirs, les Portoricains, les Mexicains ont un niveau de connaissances encore bas. Le Japon étant un pays ethniquement homogène, l'éducation y est menée avec succès ${ }^{13}$." Le mépris racial exprimé à l'encontre des Noirs, des Portoricains et des Mexicains souleva de vives critiques de la part des États-Unis et Yasuhiro Nakasone dut leur présenter des excuses. Par contre, concernant la rhétorique de l'“homogénéité ethnique du pays", excepté les critiques venues des Aïnous (premiers habitants du Japon), personne n'a critiqué cette déclaration. Le "pays ethniquement homogène", fondé sur un groupe ethnique partageant une langue et un mode de vie communs, tel qu'il est formulé par Yasuhiro Nakasone, s'oppose à l'État-nation à la française fondé sur un peuple présenté comme abstrait ${ }^{14}$. Il ne s'agit pas de prôner simplement la valeur d'une homogénéité culturelle, mais aussi une certaine pureté ethnique (niant la présence de minorités au sein même de la population japonaise) qui risque de déboucher, du moins théoriquement, sur le concept d'épuration.

Bien que, à la suite de Yasuhiro Nakasone, d'autres hommes politiques, pour la plupart du Parti libéral démocrate, aient fait des déclarations semblables, 
aucun homme politique n'a pris la responsabilité de pointer du doigt le problème. La principale raison est à chercher du côté de la pression assimilationniste envers les colonisés qui a été particulièrement forte.

L'assimilation des minorités ethniques était une étape espérée avant qu'elles fassent partie de la communauté des "Japonais authentiques". Autrement dit, l'assimilation n'était pas désirée dans le but de rejeter ou de discriminer une culture différente, mais pour faire de vrais compatriotes solidaires. L'assimilation remplit une double fonction : une fonction inclusive qui valorise une union forte au sein de la collectivité et une fonction exclusive qui définit les limites de l'appartenance citoyenne.

\section{Les composantes sociales de la xénophobie au Japon}

Une enquête menée dans la ville de Nishinomiya auprès d'hommes et de femmes de 20 à 70 ans permet de mettre en évidence les principaux facteurs contemporains de la xénophobie ainsi que leur évolution. L'échantillonnage s'est fait à partir de la liste des électeurs, en prenant en compte les circonscriptions et les générations. De la sélection de 500 personnes sont restées 430 personnes après diverses vérifications. Nous avons obtenu 178 réponses (41,4 \%). Nishinomiya est une ville d'environ 
480000 habitants située entre les deux grandes villes d'Osaka et de Kobe. Pratique, raffinée, c'est une zone de résidence prisée qui compte plusieurs ensembles immobiliers de haut standing et dont la population est d'un niveau social relativement élevé ${ }^{15}$. Au niveau politique, il s'agit d'une ville qui a tendance à choisir des représentants de partis progressistes.

Nous avons retenu un certain nombre de variables qui tendent à influer sur la xénophobie. La première est celle de la valorisation de l'assimilation. Ceux qui sont assimilés et intégrés dans la société sont idéalisés et les problèmes des autres sont rendus invisibles, ce qui renforce l'idée que l'assimilation est positive aussi pour les étrangers.

La deuxième concerne la "tendance sédentaire". $\mathrm{Au}$ Japon, on désigne les comportements xénophobes causés par un environnement de vie fermé et constant par l'expression "mentalité insulaire". En outre, il est courant de constater combien une culture agricole attachée à la terre tend à considérer l'étranger comme un ennemi.

Troisièmement, nous avons retenu une variable dite de "revendication punitive". On s'accorde à dire qu'à notre époque de "modernité tardive ${ }^{16 "}$ ", les sans domicile fixe et les délinquants/criminels, perçus comme des figures de l'Autre, ont souvent fait l'objet de rejet. C'est pourquoi nous avons sélectionné, parmi les variables de cette analyse, la revendication punitive qui se manifeste à l'égard des délinquants/criminels, ce qui permet d'évaluer le degré de tolérance à l'égard de l'Autre.

Quatrièmement, le concept de confiance généralisée a été avancé au Japon par le psychosociologue Toshio Yamagishi ${ }^{17}$ et il a été repris dans des travaux sur les réseaux sociaux qui ont donné des résultats quant à la détermination de comportements

Manifestant appartenant au mouvement raciste Zaitoku-kai. (c) Camille Millerand

xénophobes. Cinquièmement, beaucoup de travaux ont souligné les liens entre les comportements discriminants à l'égard des minorités et les situations d'oppression psychologique à commencer par les travaux sur le "downward comparison prin-

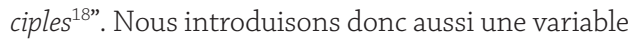
mesurant les sentiments d'“exclusion sociale", souvent considérée comme un problème majeur dans la "modernité tardive". La variable suivante est celle de l'"orientation politique" : on observe au Japon un mouvement similaire au Tea Party, une avancée du néolibéralisme assortie d'un renforcement de la xénophobie. Nous avons ici eu recours aux indices de comportement politique des enquêtes internationales sur les systèmes de valeurs.

Enfin, nous avons croisé les variables présentées ci-dessus avec un certain nombre d'autres dont les variables d'âge et de statut social (nombre d'années d'études/classes ouvrières ou non). On a observé des liens entre ces variables et la xénophobie dans de précédents travaux au Japon ${ }^{19}$. En outre, des 
recherches japonaises soulignent la relation entre la diversification des réseaux sociaux et la xénopho$b e^{20}$, nous avons donc aussi utilisé comme variable la fréquence des contacts avec des étrangers.

\section{Quatre voies de construction des sentiments xénophobes}

Notre méthodologie, que nous ne développons pas dans le présent article, est celle d'une analyse selon les modèles à équations structurelles (présentés sous la forme d'un diagramme des causalités ou chemin de causes). Cette analyse met en évidence que les facteurs les

L'idée que "les travailleurs

précaires entretiennent

la xénophobie" n'est pas

pertinente au Japon. Cela est,

selon nous, lié à l'histoire

des Coréens du Japon et à une

spécificité de la xénophobie

dans ce pays. plus influents sont la valorisation de l'assimilation et la revendication punitive. Les facteurs de moindre influence sont l'exclusion sociale, puis le néolibéralisme, la confiance généralisée et la tendance sédentaire. L'analyse des résultats permet d'aller plus loin et, tout d'abord, de rappeler qu'il s'agit d'une réalité stratifiée, d'une juxtaposition de diverses formes de xénophobie. Nous avons ainsi pu mettre en évidence quatre principales voies de construction des sentiments xénophobes.

La première découle de l'association de deux variables, à savoir la valorisation de l'assimilation et la tendance sédentaire, des inclinations sociales qui varient elles-mêmes en fonction de lâge. La variable de l'âge mêle trois types d'effets : l'effet d'âge, l'effet de période et l'effet de génération. Dans le cas de notre analyse de données en coupes transversales, il n'est pas possible de distinguer les différents effets. S'il s'agit surtout d'effets de période et de génération, et s'il n'y a pas de changement de contexte radical ${ }^{21}$, on peut s'attendre à ce que la xénophobie diminue naturellement à l'avenir. Par contre, s'il s'agit d'un effet d'âge, et que cet effet est central dans les effets globaux, la xénophobie continuera de se renouveler.

$\mathrm{Au}$ Japon, par sa fonction intégratrice, l'assimilation est considérée comme vertueuse. Même avec des jeunes, le seul fait d'évoquer les "aspects indésirables de l'assimilation" provoque régulièrement de l'étonnement, voire de vives réactions. Les résultats de l'enquête suggèrent que la valorisation de l'assimilation au Japon engendre concrètement une différenciation et l'exclusion des étrangers tout en étant considérée comme un dispositif légitime pour l'intégration. Tant que l'apologie de l'assimilation ne sera pas déconstruite, la xénophobie qui en découle conservera des fondements solides.

La deuxième se caractérise par la revendication punitive, l'exclusion sociale et à nouveau la tendance sédentaire qui varient selon la fréquence des contacts avec les étrangers. Les résultats de l'analyse ont montré que moins une personne entretient de rapports avec un étranger et plus elle a tendance à exprimer de la xénophobie. Ce qui signifie que la recherche permanente de l'entre-soi aboutit à la fermeture d'esprit et favorise l'émergence de sentiments xénophobes. Mais, comme dans d'autres pays, la xénophobie apparaît plus forte dans les quartiers où vivent de nombreux étrangers ${ }^{22}$, toutefois, dans les quartiers où habitent depuis longtemps de nombreux Coréens du Japon, on 
observe au contraire un niveau bas de xénophobie ${ }^{23}$. Cela laisse supposer que la présence étrangère de proximité et de longue date peut faire baisser la xénophobie. Là se trouvent les enjeux de toute initiative en faveur des échanges entre des cultures différentes, comme les cours de coréen, les séminaires sur les droits de l'homme, les cours de cuisine brésilienne, etc. Si l'impact de ces activités peut paraître à première vue limité à un niveau très local, elles contribuent en fait à l'enracinement du multiculturalisme dans le pays.

La troisième dépend de l'exclusion sociale et de l'inclination à la confiance généralisée qui varient en fonction des couches sociales. Alors qu'en Europe beaucoup de travaux pionniers ont utilisé la variable "couche sociale", dans le cas du Japon il n'y a pas, ou extrêmement peu, de données indiquant un effet direct de la variable "couche sociale" sur la xénophobie ${ }^{24}$. L'idée que "les travailleurs précaires entretiennent la xénophobie" n'est pas pertinente au Japon. Cela est, selon nous, lié à l'histoire des Coréens du Japon et à une spécificité de la xénophobie dans ce pays.Toutefois, notre analyse a mis en évidence des effets indirects relativement importants de la variable "couche sociale" sur la xénophobie.

De façon très classique, on observe que l'expérience de sa propre exclusion est une source de xénophobie. Le cas des membres des groupes xénophobes de droite décrit pas Kôichi Yasuda relève en partie de cette analyse ${ }^{25}$. La période de stagnation économique qui atteint maintenant près de vingt ans et l'impact d'une politique de l'emploi néolibérale expliquent l'augmentation des inégalités entre les différentes couches sociales au Japon. Si l'on considère le nombre croissant de pauvres, cette source de xénophobie devrait se confirmer et s'amplifier dans les années à venir. En effet, dans ses travaux de 1999, Kunisuke Hamada n'observait pas d'impact de l'appartenance sociale sur la xénophobie, contrairement à ce qu'ont révélé les données de 2005.
Enfin, la quatrième voie de construction des sentiments xénophobes découle des orientations politiques. Ce que nous nommons "néolibéralisme" dans notre étude correspond au souhait d'une "société assurant un minimum de services publics et de bien-être et imposant un minimum de taxes". Un tel point de vue politique est une source importante de xénophobie.Cepositionnementpolitique est en corrélation avec la popularité de la droite libertaire représentée entre autres
Il s'agit d'un "état d'esprit du moment" sans racines dans le contexte social, d'une réaction émotionnelle qui se répand dans la société japonaise sans qu'on puisse l'expliquer par des facteurs structurels. par le succès du parti Osaka RestorationAssociationdumaired'Osaka,TôruHashimoto, ou du gouverneur de Tokyo (au moment de l'enquête), Ishihara Shintarô. Cela n'est pas sans rappeler la xénophobie alimentée par le mouvement Tea Party aux États-Unis.

Cette idéologie politique n'a pas de relations avec les caractéristiques sociales des individus. Il s'agit d'un "état d'esprit du moment" sans racines dans le contexte social, d'une réaction émotionnelle qui se répand dans la société japonaise sans qu’on puisse l'expliquer par des facteurs structurels.

Notre enquête menée à Nishinomiya a permis de renouveler le débat sur la xénophobie au Japon en confirmant certaines analyses et en mettant en relief de nouvelles tendances. Elle confirme que les sources de la xénophobie sont diverses et stratifiées. Elle confirme aussi la corrélation positive entre les contacts avec les étrangers et la baisse de la xénophobie. Ellepermet d'affinerl'importance des effets d'âge qui doivent être compris en relation avec le discours dominant. Elle permet, enfin, de mettre en évidence le caractère conjoncturel de la corrélation entre idéologie néolibérale et xénophobie, ainsi que l'émergence au Japon des liens entre "couches sociales" et xénophobie qui n'existaient pas jusqu’alors.

\section{Traduit du japonais par Chikako Mori} et Hélène Le Bail 